\title{
La duchesse Constance de Bretagne et le gouvernement en héritage à la fin du XII ${ }^{\mathrm{e}}$ siècle
}

The Duchess Constance of Brittany and the inheritance of government in the late twelfth century

\section{Élodie Chaudet}

\section{(2) OpenEdition}

\section{Journals}

Édition électronique

URL : https://journals.openedition.org/abpo/4349

DOI : $10.4000 / a b p o .4349$

ISBN : 978-2-7535-7783-1

ISSN : 2108-6443

\section{Éditeur}

Presses universitaires de Rennes

\section{Édition imprimée}

Date de publication : 20 mars 2019

Pagination : $31-52$

ISBN : 978-2-7535-7782-4

ISSN : 0399-0826

\section{Référence électronique}

Élodie Chaudet, «La duchesse Constance de Bretagne et le gouvernement en héritage à la fin du х॥" siècle ", Annales de Bretagne et des Pays de l'Ouest [En ligne], 126-1 | 2019, mis en ligne le 01 janvier 2022, consulté le 08 janvier 2022. URL : http://journals.openedition.org/abpo/4349 ; DOI : https:// doi.org/10.4000/abpo.4349 


\title{
La duchesse Constance de Bretagne et le gouvernement en héritage à la fin du XII ${ }^{\mathrm{e}}$ siècle
}

\author{
Élodie CHAUDET \\ Doctorante en histoire médiévale, université d'Angers, TEMOS
}

En $1186^{1}$, le duc de Bretagne Geoffroy II décède à la cour de Philippe Auguste (1180-1223), roi de France ${ }^{2}$. Les circonstances de sa mort ne sont pas claires, oscillant entre une fièvre et un accident de tournoi ${ }^{3}$. Le roi capétien lui accorde des funérailles grandioses, et il est enterré dans le chœur de la cathédrale Notre-Dame de Paris. Geoffroy laisse derrière lui deux filles, Aliénor (ou Éléonore) et Mathilde (probablement morte en 1189) ${ }^{4}$, ainsi que son épouse Constance de Bretagne (1161-1201), qui est enceinte. Le 29 mars 1187, un fils naît à Nantes; il est prénommé

1. La datation se fait en Nouveau Style dans tout l'article.

2. Selon Yannick Hillion, la présence de Geoffroy, fils d'Henri II et d'Aliénor d'Aquitaine, s'explique par le fait qu'il organisait un complot visant à récupérer des territoires frontaliers de la Bretagne (Hitlion, Yannick, " La Bretagne et la rivalité Capétiens-Plantagenêts. Un exemple : la duchesse Constance (1186-1202)", Annales de Bretagne et des pays de l'Ouest, 1985, vol. 92, n 2, p. 111-144, p. 112).

3. Borgnis-DesBoRdes, Éric, Constance de Bretagne (1161-1201). Une duchesse face à Richard Coeur de Lion et Jean sans Terre, Fouesnant, Yoran embanner, 2018, p. 217-221. L'ouvrage, paru tout récemment, retrace la vie de Constance de Bretagne en appuyant son étude sur les sources narratives. En ce qui concerne la mort de Geoffroy II, l'auteur explique que la sépulture chrétienne était à cette époque interdite aux chevaliers morts de piétinements. La présence d'un bas-relief, qui a peut-être été commandé par Constance, dans l'abbaye de Saint-Gildas-de-Rhuys représentant deux cavaliers lors d'un tournoi tend à conforter l'hypothèse de l'accident de tournoi. Nous avons appris l'existence d'un travail universitaire que nous n'avons pas eu l'occasion de consulter : SoHIER, Mari-Anna, Étude des actes de la duchesse Constance et de sa famille, mémoire de maîtrise, université de Brest, 2003.

4. Jones, Michael, «La vie familiale de la duchesse Constance : le témoignage des chartes ", dans LE MENN, Gwennolé (dir.), Bretagne et pays celtiques : langues, histoire, civilisation : mélanges offerts à la mémoire de Léon Fleuriot, Presses universitaires de Rennes, Saint-Brieuc, 1992, p. 349-361, p. 352. 
Arthur $^{5}$. La mort du duc entraîne une nouvelle période de gouvernement, assurée par Constance ${ }^{6}$. Fille du duc de Bretagne Conan IV, elle a épousé Geoffroy Plantagenêt, troisième fils d'Henri II Plantagenêt (1154-1189) et d'Aliénor d'Aquitaine, en 1166. Par cette alliance, Geoffroy est devenu duc de Bretagne en 1181. Il convient dès lors de s'interroger sur le statut de Constance de Bretagne. Gouverne-t-elle en tant qu'épouse de duc défunt, en tant que fille de duc défunt, en tant que mère de duc en devenir? Comment conçoit-elle son propre gouvernement? En quelle qualité se présente-t-elle dans les actes? Sa situation est-elle reconnue au sein du duché? Les rois anglais et capétien reconnaissent-ils son gouvernement?

Les questions soulevées par la période du gouvernement de Constance de Bretagne sont intimement liées à son sexe ${ }^{7}$. Femme au pouvoir, sa situation est originale puisque la gouvernance du duché est traditionnellement assurée par un homme ${ }^{8}$. L'originalité de cette configuration impose d'étudier la mise en place du gouvernement de Constance et sa réception au sein du duché de Bretagne. La question de la reconnaissance de la détention de l'autorité ducale est primordiale pour évaluer la légitimité de la position de Constance.

L'étude porte sur l'analyse du corpus des actes émis par Constance, édité par Michael Jones et Judith Everard ${ }^{9}$. Les soixante-dix-sept chartes émises par Constance de Bretagne ont été comparées à d'autres cor-

5. Borgnis-Desbordes, Éric, Arthur de Bretagne, 1187-1203 : l'espoir breton assassiné, Fouesnant, Yoran embann, 2012, p. 164.

6. La période du gouvernement de Constance de Bretagne a fait l'objet d'un mémoire de master, intitulé Constance de Bretagne (1186-1201), femme de pouvoir, femme au pouvoir en Bretagne au XII siècle, réalisé en 2014-2015 sous la direction de Carole Avignon.

7. L'étude du rôle des femmes dans la succession ducale a été réalisée sur trois générations par Mélissa Pollock, qui s'est concentrée sur Constance, sa mère Marguerite d'Écosse, et sa grand-mère Berthe (Pollock, Mélissa, " Duchesses and devils : the Breton succession crisis [1148-1189] ", French History, vol. 3, no 2, 2009/06, p. 149-170, [https:// academic.oup.com/fh/article/23/2/149/633495\#10763735] consulté le 13/12/2018).

8. Traditionnellement mais pas exclusivement. Amy Livingstone a mis en lumière la figure d'Ermengarde de Bretagne, duchesse ayant exercé personnellement et conjointement le pouvoir ducal durant ce même Moyen Âge central (LivingsTone, Amy, "Extraordinairement ordinaire : Ermengarde de Bretagne, femmes de l'aristocratie et pouvoir en France au Moyen-Âge, v. 1090-1135 ", Annales de Bretagne et des pays de l'Ouest, n 1, 2014/121-1, p. 7-25).

9. JONES, Michael et EVERARD Judith Ann, The charters of Duchess Constance of Britanny and her family: 1171-1221, Woodbridge Rochester, Boydell Press, 1999. Pour la période, il existe également un certain nombre de sources narratives, chroniques et poèmes. Pour le règne d'Henri II, nous pouvons mentionner La Gesta Regis Henrici Secondi de Bernard de Peterborough, l'Historia Anglicana de Guillaume de Newburgh, le De Rebus gestis Anglorum de Guillaume de Malmesbury, la chronique de Robert de Torigni (abbé du Mont SaintMichel entre 1145 et 1189), le récit de Raoul de Diceto et le poème Draco Normannicus, probablement écrit vers 1168 par Étienne de Rouen. Pour le règne de Richard Cœur de Lion, retenons le récit de Raoul de Coggeshale : Chronico Anglicanum. Philippe Auguste nous est enfin connu grâce à l'Histoire de la vie et des gestes de Philippe Auguste, rédigé par Guillaume le Breton, qui continue l'œuvre de Rigord, et le poème du même auteur intitulé La Philippide. 
pus d'actes émis par d'autres ducs et duchesses de Bretagne ${ }^{10}$ : prédécesseurs $^{11}$, contemporains et quasi-contemporains ${ }^{12}$, et successeurs ${ }^{13}$ de Constance de Bretagne. L'intégralité des ducs et duchesses intégrés à l'étude n'était certes pas connue de Constance. En revanche, les pratiques de cette dernière ont pu influencer celles de ses successeurs. En plaçant Constance dans une chronologie qui dépasse son simple règne, et qui s'étend avant et après ce dernier, nous pouvons déterminer si la duchesse s'inscrit dans l'évolution globale des pratiques gouvernementales ducales, ou bien si elle apparaît comme un élément dont le gouvernement est entièrement original, que ce soit par rapport à ses prédécesseurs ou à ses successeurs. La confrontation des différents corpus étudiés a permis de dégager des éléments de compréhension quant à l'exercice du pouvoir par Constance de Bretagne.

Constance de Bretagne gouverne tel un duc, produisant des chartes qui lui permettent d'administrer son duché. Si la régence n'est pas encore institutionnalisée, les absences et minorités de rois font naître les premières expériences d'interrègne. Les chartes de Constance ne mentionnent pourtant pas une quelconque activité d'interrègne, puisqu'elle s'y présente comme duchesse de Bretagne et comtesse de Richemont, fille du comte

10. Il est à noter que la sélection de corpus concernant cette démarche comparative s'est faite selon les recueils édités à ce jour.

11. Pour la période antérieure à la seconde moitié du XII ${ }^{\mathrm{e}}$ siècle, nous disposons de la thèse d'Hubert Guillotel (Actes des ducs de Bretagne, 944-1148, Rennes, Presses universitaires de Rennes, 2014), qui a édité les actes des ducs de Bretagne de 944 à 1148 (175 actes). Cette période correspond aux règnes de Bérenger, Juhel Bérenger, Conan I ${ }^{\mathrm{er}}$, Geoffroy I ${ }^{\text {er }}$, Alain III, Conan II, Hoël, Alain IV et Conan III, soit neuf ducs. En revanche, nous ne disposons pas de recueils édités pour les ducs et duchesses ayant gouverné la Bretagne de 1148 à 1166 (Eudon de Porhoët et Berthe, puis Conan IV).

12. L'édition de Judith Everard et Michael Jones propose également les corpus de chartes de la mère de Constance, Marguerite d'Écosse (7 chartes), de ses trois époux (Geoffroy Plantagenêt, avec 30 chartes, Ranulf de Chester, avec 24 chartes, et Guy de Thouars, avec 31 chartes) et de trois de ses enfants (Arthur, avec 23 chartes, Alix, avec 25 chartes, et Aliénor - nous n'avons pas retenu cette dernière car son corpus ne se compose que de deux chartes).

13. Il s'agit des recueils d'actes de Pierre de Dreux (1213-1237, 120 actes) et de Jean I ${ }^{\text {er }}$ (1237-1286, 176 actes) pour le XIII ${ }^{\mathrm{e}}$ siècle, ces deux recueils ayant été publiés par Marjolaine Lémeillat (Actes de Pierre de Dreux, duc de Bretagne (1213-1237), Rennes, Presses universitaires de Rennes, 2013, et Actes de Jean I Ir, duc de Bretagne (1237-1286), Rennes, Presses universitaires de Rennes, 2014). Concernant le XIV ${ }^{\mathrm{e}}$ siècle, nous avons utilisé les recueils des actes de Charles de Blois et de Jeanne de Penthièvre (1341-1364, 301 actes) et de Jean IV (1357-1382, 438 actes), tous deux édités par Michael Jones (Recueil des actes de Charles de Blois et de Jeanne de Penthièvre, duc et duchesse de Bretagne, 13411364, Rennes, Presses universitaires de Rennes, 1996 et Recueil des actes de Jean IV, duc de Bretagne, (1347-1382), Paris, Klincksieck, 1980). Il est à noter que pour le recueil de Charles de Blois et de Jeanne de Penthièvre, les actes sont divisés en deux périodes. La première est celle indiquée dans le titre, donc de 1341 à 1364 (soit jusqu'à la mort de Charles de Blois). Une seconde période, partant de 1364 et se prolongeant jusqu'en 1384, concerne les actes émis par Jeanne de Penthièvre après le décès de son époux (62 actes). Concernant le recueil de Jean IV, il ne s'agit que d'une partie de son règne, puisque celui-ci a été duc de 1365 à 1399. 
Conan. Titres et filiation ne sont là que pour rappeler sa légitimité et justifier son statut de gouvernante. Si sa position d'héritière est implicitement mentionnée par la très fréquente évocation de son père dans sa titulature (voir tableau en annexe), elle ne se présente jamais, à partir de 1186-1187, comme étant l'épouse de son défunt mari le duc Geoffroy, ni comme la mère du futur duc de Bretagne, Arthur. C'est sa propre filiation qu'elle met en avant : elle est fille de duc breton, ce qui fait d'elle la duchesse.

Constance adopte ainsi une stratégie propre de démonstration de sa légitimité. Les instruments de son gouvernement sont autant de moyens pour elle d'affirmer avec fermeté son statut. Cependant, il existe des divergences entre la stratégie évoquée et la pratique de Constance dans les faits, puisqu'elle semble implicitement adopter un comportement d'interrègne. Enfin, le sexe de Constance constitue, pour les souverains anglais et français, une opportunité de placer la Bretagne sous leur tutelle directe ou indirecte.

\section{Une femme à la tête du duché de Bretagne}

L'étude du gouvernement de Constance de Bretagne s'inscrit dans son contexte, marqué par l'affrontement opposant les Plantagenêts aux Capétiens. La mort du duc Conan III (1119-1148) entraîne une grave crise de succession. Ce dernier a en effet déshérité son fils Hoël au profit de sa fille Berthe, épouse en premières noces d'Alain le Noir, comte de Richemont et héritier du comté de Penthièvre, avec qui elle a eu deux enfants : Conan et Constance ${ }^{14}$. Berthe a ensuite épousé Eudon de Porhoët, qui assure le gouvernement du duché tandis qu'Hoël a été écarté de la succession ${ }^{15}$.

Conan, issu du premier mariage de Berthe, se voit refuser le pouvoir par Eudon en 1154. Vaincu par son beau-père, Conan quitte la Bretagne et obtient le comté de Richemont du roi d'Angleterre, Henri II, qui devient son seigneur ${ }^{16}$. Grâce au soutien de ce dernier, Conan conquiert le duché et devient duc sous le nom de Conan IV. Le comté de Nantes reste cependant dominé par Hoël, jusqu'à ce que les Nantais le chassent pour appeler Geoffroy, frère d'Henri II, à sa place. À la mort de Geoffroy, le roi d'Angleterre se déclare son héritier et obtient la cession solennelle du comté de Nantes par Conan IV après plusieurs menaces ${ }^{17}$. L'événement permet de souligner la fragilité de l'indépendance bretonne ${ }^{18}$, d'autant plus qu'à l'inté-

14. Borgnis-Desbordes, Éric, Constance de Bretagne..., op. cit., p. 22.

15. ChedeVILle, André et Tonnerre Noël Yves, La Bretagne féodale, XI ${ }^{e}$-XIII $I^{e}$ siècle, Rennes, Ouest-France, 1987, p. 84.

16. Ibid., p. 85 .

17. Le PATOuREL, John, "Henri II Plantagenêt et la Bretagne ", dans Feudal empires : Norman and Plantagenet, s.l., Hambledon press, 1984, p. 99-116, p. 100-101.

18. Selon Noël Yves Tonnerre, il s'agit de " la première étape dans la soumission du duché au pouvoir angevin " (Chedeville, André et TonnerRe, Noël Yves, La Bretagne féodale..., op. cit., p. 86). 
rieur même du duché, le mécontentement des barons amène à plusieurs révoltes ${ }^{19}$.

Cette instabilité pousse Henri II à écarter Conan IV du gouvernement du duché. En 1166, le projet de mariage de Geoffroy et de Constance entraîne l'abdication de Conan IV ${ }^{20}$. L'administration du duché est assurée par le roi d'Angleterre jusqu'à la majorité des fiancés ${ }^{21}$. La Bretagne est ainsi prise en main par Henri II, tandis que Conan IV ne garde que le comté de Guingamp ${ }^{22}$, son autorité sur la baronnie de Tréguier et sur l'honneur de Richemont, et les titres de duc de Bretagne et de comte de Richemont ${ }^{23}$. Le souverain Plantagenêt dote le duché d'une véritable administration princière : les fonctions des officiers ducaux sont plus clairement définies, la chancellerie se structure et l'office de sénéchal de Bretagne est créé. Le gouvernement indirect d'Henri II sur la Bretagne constitue paradoxalement un moment clé dans l'unification de la Bretagne sous l'autorité d'un seul ${ }^{24}$.

Le roi anglais doit cependant à son tour faire face à plusieurs révoltes ${ }^{25}$. Son gouvernement étant une période transitoire, le rôle joué par Geoffroy va croissant : il est notamment associé à l'administration de son père ${ }^{26}$. L'accession de Geoffroy à la tête du duché (hormis le comté de Nantes retenu par Henri II) se fait alors qu'il a 23 ans, en 1181, au moment où son mariage avec Constance est enfin célébré. Geoffroy Plantagenêt, en

19. Raoul de Fougères se rebelle au début des années 1160 , ce qui conduit à la destruction du château de Fougères (CHEDEville, André et TonNERre, Noël Yves, La Bretagne féodale..., op. cit., p. 87-88).

20. Selon Martin Aurell, l'abdication de Conan IV est motivée par deux raisons. D’une part, la Bretagne se retrouve ainsi intégralement conservée, et non pas amputée, et d'autre part elle garde également son indépendance (AURELL, Martin, L'empire des Plantagenêt, 1154-1224, Paris, Perrin, 2004, p. 218).

21. L'existence de William, frère de Constance, est attestée par deux chartes, dans lesquelles il est désigné comme "Willelmo clerico, fratre Comitisse " (JonEs, Michael et EVERARD, Judith Ann, The charters..., op. cit., C45, p. 75-76 et A16, p. 128-129). Il semble qu'il n'a jamais été envisagé que ce fils succède à Conan IV, ce qui pourrait indiquer qu'il s'agit d'un fils illégitime. Mélissa Pollock souligne que quelques décennies plus tôt, une fille, Berthe, a également été privilégiée pour la succession par son père Conan III au détriment, d'un fils, Hoël (Pollock, Mélissa, " Duchesses and devils... ", art. cité).

22. ChÉdeville, André et Tonnerre, Noël Yves, La Bretagne féodale..., op. cit., p. 88.

23. EVERARD, Judith Ann, Brittany and the Angevins : province and empire, 1158-1203, New York, Cambridge University Press, 2000, p. 44.

24. ChÉDEVILLE, André et TonNERre, Noël Yves, La Bretagne féodale ..., op. cit., p. 104-106.

25. Selon Fanny Madeline, ces révoltes ne constituent pas une véritable source d'instabilité pour le duché, puisque ce sont des initiatives individuelles (avec toujours les mêmes individus). En effet, elle souligne que les seigneurs de la marche bretonne sont bien intégrés dans un espace anglo-normand, d'autant plus qu'ils ont des possessions en Normandie et en Angleterre (depuis la conquête de l'Angleterre en 1066). Bien au contraire, selon elle, il s'agirait d'une période de renforcement de l'autorité ducale (MADELINE, Fanny, Les Plantagenêts et leur empire : construire un territoire politique, Rennes, Presses universitaires de Rennes, 2014, p. 179).

26. Si Geoffroy est associé à l'administration de son père, c'est, selon Judith Everard, pour légitimer l'action de ce dernier et se doter de précédents pour son futur gouvernement (EvERARD, Judith Ann, Brittany and the Angevins..., op. cit., p. 94). 
tant qu'époux de Constance, héritière de duché de Bretagne, est considéré comme légitime. Il obtient ainsi le soutien de grands seigneurs ${ }^{27}$. Geoffroy, bien qu'issu de la dynastie d'origine angevine, parvient à se concilier les barons grâce à son union avec Constance, ainsi que par ses méthodes de gouvernement. D'une part, il réside en Bretagne, et d'autre part, il tend à imiter les anciens ducs originaires du duché ${ }^{28}$. Geoffroy est ainsi intégré et accepté par les barons. Des tensions entre Geoffroy et son père apparaissent au cours des années $1182-1183^{29}$. Le décès de l'héritier du roi d'Angleterre, Henri le Jeune, change la succession, et l'attribution des différents territoires angevins est modifiée. Geoffroy passe de troisième fils dans la ligne de succession, à deuxième ${ }^{30}$, tandis que Richard, sans enfant, devient le premier. Le duc de Bretagne lorgne alors sur l'Anjou, qui revient finalement à Richard. Henri II restitue des terres à Geoffroy ${ }^{31}$, qui gouverne ainsi l'intégralité du duché de Bretagne ${ }^{32}$, sans qu'il soit possible d'écarter l'influence du souverain anglais.

Lorsque Constance de Bretagne parvient au pouvoir, le duché en tant qu'entité féodale à part entière est donc menacé et la position du gouvernant du duché, au sein de l'espace Plantagenêt, apparaît fragile. Pourtant, les actes émis par la duchesse témoignent de son exercice réel du pouvoir sur le duché de Bretagne, puisqu'elle s'en sert comme outils de gouvernement $^{33}$.

27. Comme le souligne Martin Aurell, « la noblesse bretonne est attachée à l'unification du duché sous une autorité locale, une lignée propre et exclusive " (AuRELL, Martin, L'empire des Plantagenêt..., op. cit., p. 220).

28. JonEs, Michael et EvERARD, Judith Ann, The charters..., op. cit., p. 1. Geoffroy s'associe à "l'identité bretonne " de Constance, ce qui lui permet de ne pas apparaître comme un prince étranger (POLlock, Mélissa, "Duchesses and devils... ", art. cité).

29. En 1183, Geoffroy se rebelle avec son aîné, Henri le Jeune, mais la révolte tourne court avec le décès de ce dernier. En guise de punition, Henri II confisque des fortifications et des châteaux appartenant à Geoffroy (EvERARD, Judith Ann, Brittany and the Angevins..., op. cit., p. 132-134).

30. Ibid., p. 138.

31. En 1183, il débute par l'honneur de Richmond, qu'il n'avait pas cédé en 1181; puis, en 1185, il lui laisse le comté de Nantes (JONES, Michael et EvERARD, Judith Ann, The charters..., op. cit., p. 1).

32. La période du règne de Geoffroy sur la Bretagne est l'objet de débats concernant l'intervention, ou non, d'Henri II dans ce gouvernement. De nombreux historiens considèrent que le roi anglais exerce sur la Bretagne une " tutelle camouflée " (TouraulT, Philippe, Les ducs et duchesses de Bretagne : $X^{e}-X v f^{e}$ siècle, Paris, Perrin, 2009, p. 100). Judith Everard affirme en revanche que le monarque anglais n'intervient que dans certains cas précis, motivés par des raisons spécifiques, qui sont les suivantes : lorsque l'affaire en question a une chronologie qui débute avant 1181, ou lorsque l'affaire est diplomatique et qu'elle concerne le roi de France. Quant à son intervention militaire datée de 1183, la raison en est inconnue pour l'auteure (EVERARD, Judith Ann, Brittany and the Angevins..., op. cit., p. 97-98).

33. Constance a émis trois types de chartes : des documents économiques relatifs aux sources de revenus et à la propriété $(55$ chartes : $\mathrm{C} 1 ; \mathrm{C} 2 ; \mathrm{C} 3 ; \mathrm{C} 5 ; \mathrm{C} 7$; C8; C9; C10; C12 ; C14; C15; C16; C18; C19; C22; C23; C24; C27; C29; C30; C32; C33; C36; C37; C39; C41; C42; C43; C44; C45; C46; C48; C51; C52; C53; C54; C55; C57; C58; C59; C60; C61; C62; $\mathrm{C} 63 ; \mathrm{C} 64 ; \mathrm{C} 65 ; \mathrm{C} 66 ; \mathrm{C} 67 ; \mathrm{C} 68 ; \mathrm{C} 69 ; \mathrm{C} 70 ; \mathrm{C} 71 ; \mathrm{C} 73 ; \mathrm{C} 75 ; \mathrm{C} 77)$, des documents personnels 
Sur soixante-dix-sept chartes, soixante et une ont été émises par Constance seule ${ }^{34}$. Cette pratique d'émission solitaire souligne que l'exercice du pouvoir par Constance s'est effectué sans tutelle. Elle a gouverné seule, certes entourée de conseillers, mais ayant le choix de la décision finale et de l'application effective des décisions. La plupart des ducs de Bretagne évoqués émettent seuls la majorité des actes qui nous sont parvenus. Cette majorité oscille entre environ la moitié à plus de $90 \%$ du total des corpus étudiés. Il s'agit d'une affirmation de la détention du pouvoir dans les mains d'une seule personne : le duc de Bretagne. Cette autorité ducale n'a pas besoin, dans la majorité des cas, de s'appuyer sur un tiers pour légitimer sa production d'actes. Cette production s'accroît au fil des siècles, sans pour autant renverser cette tendance. Constance n'y déroge pas. Étant détentrice de l'autorité ducale, quasiment $80 \%$ des chartes qu'elle produit sont émises par elle-seule. Elle s'inscrit donc dans une pratique gouvernementale traditionnelle. Il s'agit d'une preuve de son plein exercice de l'autorité ducale.

Pareillement à ses prédécesseurs et à ses successeurs, Constance de Bretagne privilégie les deux principales villes du duché pour l'émission de ses chartes : Nantes $\left(10\right.$ chartes $\left.^{35}\right)$ et Rennes $\left(6\right.$ chartes $\left.^{36}\right)$. Les autres localités d'émission des chartes sont majoritairement présentes dans les corpus des autres ducs et duchesses. Constance pratique ainsi une itinérance du pouvoir cohérente, avec une présence politique accrue dans les deux principales villes du duché ${ }^{37}$. Cet élément marque une nouvelle fois un exercice effectif du pouvoir ducal.

Les objets des chartes émises par Constance nous éclairent particulièrement sur les pratiques gouvernementales de la duchesse. Nous avons noté vingt-deux objets différents, ce qui montre la diversité des activités de Constance. Les deux objets principaux sont la confirmation ${ }^{38}$ et l'octroi ${ }^{39}$,

relatifs aux œuvres de piété et à la conjugalité (10 chartes : C4; C17; C20; C25; C31; C38; $\mathrm{C} 49 ; \mathrm{C} 72 ; \mathrm{C} 74 ; \mathrm{C} 76)$ et des documents politiques relatifs à la diplomatie et au gouvernement $(12$ chartes : $\mathrm{C} 6$; $\mathrm{C} 11 ; \mathrm{C} 13 ; \mathrm{C} 21 ; \mathrm{C} 26$; 28 ; 34 ; C35; $40 ; \mathrm{C} 47$; 50 ; C56.)

34. $\mathrm{C} 3 ; \mathrm{C} 4 ; \mathrm{C} 5 ; \mathrm{C} 11 ; \mathrm{C} 12 ; \mathrm{C} 13 ; \mathrm{C} 14 ; \mathrm{C} 15 ; \mathrm{C} 16 ; \mathrm{C} 17 ; \mathrm{C} 18 ; \mathrm{C} 19 ; \mathrm{C} 20 ; \mathrm{C} 21 ; \mathrm{C} 22 ; \mathrm{C} 23 ; \mathrm{C} 24$; $\mathrm{C} 25$; 226 ; 227 ; 28 ; C29; C30; C31; C32; C33; C34; C36; C37; C38; C39; C40; C41; C45; C47; C49; C50; C 52; C53; C54; C55; C56; C57; C58; C59; C60; C61; C62; C63; C64; C65; $\mathrm{C} 66 ; \mathrm{C} 67 ; \mathrm{C} 68 ; \mathrm{C} 69 ; \mathrm{C} 70 ; \mathrm{C} 71 ; \mathrm{C} 72 ; \mathrm{C} 73 ; \mathrm{C} 75 ; \mathrm{C} 76$.

35. C8; $\mathrm{C} 19 ; \mathrm{C} 37 ; \mathrm{C} 38 ; \mathrm{C} 41 ; \mathrm{C} 51 ; \mathrm{C} 52 ; \mathrm{C} 54 ; \mathrm{C} 66 ; \mathrm{C} 70$.

36. $\mathrm{C} 6 ; \mathrm{C} 12 ; \mathrm{C} 29 ; \mathrm{C} 30 ; \mathrm{C} 32 ; \mathrm{C} 69$.

37. Cette préférence ducale pour les villes de Rennes et de Nantes se confirme sans pour autant qu'elles soient des capitales officielles.

38. Les objets des confirmations sont : des achat, rachat et vente, des droits et franchises, une renonciation de revendication, des termes d'installation, des traités (de paix par exemple) et des octrois, dont les objets sont similaires à ceux de la note suivante.

39. Les objets des octrois sont : de l'argent (sous forme de sommes ponctuelles ou de pensions et de rentes ou encore de recettes de taxes), des bâtiments, des droits, des hommes, des produits et des terres. 
avec respectivement 28 chartes $^{40}$ et 25 chartes $^{41}$ concernées. Ces chartes s'inscrivent dans deux logiques gouvernementales, l'une de réactivation du lien féodo-vassalique entre la domina et son réseau de seigneurs, permettant à la domina en question de consolider ses appuis politiques, et l'autre de donatrice envers les "vieilles abbayes bénédictines associées à la dynastie ducale ${ }^{42}$ ". De plus, les réactivations de donations, soit des confirmations, marquent l'autorité de Constance sur les territoires concernés, lorsqu'ils sont bretons. La duchesse est ainsi sollicitée pour donner de la valeur à de précédentes donations. Les corpus postérieurs à l'existence de Constance révèlent qu'elle aussi était mentionnée, après sa mort, dans certains actes. Ces actes sont de deux sortes : les œuvres pieuses en sa mémoire et les confirmations de donations réalisées par la duchesse. Cela signifie que sa production de chartes a bien été reconnue : elle a été conservée et validée par des ducs postérieurs, qui ont réactivé le contenu de ses textes. L'autorité ducale de Constance n'est donc pas contestée de son vivant, ni même par la suite. La confirmation d'un acte émis par un prédécesseur souligne la filiation ducale entre les différents ducs et duchesses. Le duc s'autorise à confirmer et réactiver l'acte d'un duc antérieur car il se considère comme son successeur, direct ou indirect. Ainsi, la confirmation des chartes de Constance ne signifie pas seulement la reconnaissance de son pouvoir ducal, mais l'intègre également dans une chronologie de l'autorité ducale et dans la succession qui la construit. La spécificité du climat politique de son gouvernement, avec le duché sous domination Plantagenêt, et son sexe n'effacent en rien l'activité gouvernementale de Constance. N'étant pas remise en question, son autorité ne diffère pas de celle d'un duc. De même, par ces chartes, Constance nomme des officiers auxquels elles donnent des ordres ${ }^{43}$. Elle se situe donc à la tête de la hiérarchie des officiers qui permettent au pouvoir ducal d'avoir une présence déléguée au sein du duché. L'activité diplomatique transparaît également avec les traités et les chartes relatives à la paix ${ }^{44}$, impliquant parfois Richard Cœur de Lion. En outre, il est évident qu'en tant qu'entité faisant partie de l'empire Plantagenêt, la Bretagne se retrouve réduite dans ses marges de manœuvre en termes de relations étrangères. L'autorité ducale ne peut pas adopter une politique contraire à celle du monarque anglais concernant les autres puissances, sous peine d'entrer en conflit ouvert avec le souverain Plantagenêt.

Ces éléments permettent d'affirmer que Constance a réellement gouverné la Bretagne ${ }^{45}$, qu'elle a été grande décisionnaire du duché et que sa

40. C3; C4; C5; C12; C14; C15; C16; C18; C19; C23; C24; C27; C29; C30; C35; C36; C40; $\mathrm{C} 52 ; \mathrm{C} 55 ; \mathrm{C} 59 ; \mathrm{C} 60 ; \mathrm{C} 62 ; \mathrm{C} 63 ; \mathrm{C} 66 ; \mathrm{C} 67 ; \mathrm{C} 70 ; \mathrm{C} 72 ; \mathrm{C} 75$.

41. C1;C7;C9;C10;C17;C20; C22;C31;C38;C39;C41;C42;C43;C45;C48;C51;C54; $\mathrm{C} 57 ; \mathrm{C} 58 ; \mathrm{C} 61 ; \mathrm{C} 64 ; \mathrm{C} 65 ; \mathrm{C} 69 ; \mathrm{C} 71 ; \mathrm{C} 73$.

42. EvERARD, Judith Ann, Brittany and the Angevins..., op. cit., p. 150-151.

43. $\mathrm{C} 11 ; \mathrm{C} 26 ; \mathrm{C} 50$.

44. $\mathrm{C} 21 ; \mathrm{C} 34 ; \mathrm{C} 47$.

45. BOUSMAR, Éric, « Comprendre les femmes de pouvoir (500-1800) : conclusions valenciennoises ", in NAYT-DUBoIs, Armelle et SANTINELLI, Emmanuelle, Femmes de pouvoir et pou- 
position a été acceptée des Bretons. Deux facteurs expliquent cette acceptation : l'origine bretonne de Constance et sa légitimité.

\section{Une duchesse ${ }^{46}$ native du duché et légitime}

Constance de Bretagne parvient au pouvoir à la suite du décès de son époux. Il faut cependant noter que dès le vivant de Geoffroy II, elle était associée au pouvoir. Elle a ainsi émis sept chartes conjointement avec son époux avant son décès ${ }^{47}$. Elle n'était, de ce fait, pas écartée du pouvoir lors de cette période. Geoffroy détient le titre ducal et exerce le pouvoir qui l'accompagne de par son mariage avec Constance. Il ne saurait gouverner le duché sans l'appui de son épouse, héritière légitime et native de la Bretagne. Les Bretons sont attachés à leur particularisme territorial et Constance en constitue le gage pendant cette période de domination Plantagenêt. Une fois Geoffroy réellement duc de Bretagne, le duché n'est pas directement placé sous l'autorité d'Henri II. S'il est considéré comme étant un élément de l'empire Plantagenêt, il s'agit d'un territoire sous l'influence des Plantagenêts par les liens de parenté.

Après le décès de Geoffroy II, Constance gouverne en son nom ${ }^{48}$. Elle se présente dans les chartes grâce à sa titulature, comprenant le prénom, les titres de duchesse de Bretagne et de comtesse de Richemont, et l'affiliation avec Conan IV, qui est largement majoritaire, puisqu'elle concerne un peu moins de la moitié des chartes (voir tableau en annexe). Il s'agit des quatre éléments récurrents de la titulature. Les titulatures variantes se composent de ces éléments, mais ne les possèdent pas tous les quatre.

Les deux titres de duchesse et comtesse désignent l'épouse du duc, et il s'agit d'une évolution des termes. Avec l'hérédité, les épouses des princes peuvent devenir leurs consors, et c'est ainsi qu'apparaissent des titres féminins correspondant aux titres masculins, tels ducissa, comitissa. La femme du seigneur est considérée comme la domina de tous les vassaux de son époux ${ }^{49}$. Dans le cas de Constance, nous avons bien une ducissa reconnue comme épouse du duc et domina de ses vassaux. Son époux est duc par son mariage avec Constance. Elle n'est donc pas une simple consors, mais

voir des femmes dans l'Occident médiéval et moderne, Valenciennes, Presses universitaires de Valenciennes, 2009, p. 483-499. L'auteur définit les femmes de pouvoir comme celles réussissant effectivement à s'approprier et à exercer le pouvoir (p. 490).

46. Un ouvrage de Laurence Moal sur les duchesses de Bretagne durant la seconde moitié du Moyen Âge va prochainement paraître aux Presses universitaires de Rennes.

47. $\mathrm{C} 1$; $\mathrm{C} 2$; $\mathrm{C} 6$; $\mathrm{C} 7$; C8; C9; C10.

48. Melissa Pollock affirme que la mort de son époux aurait dû donner à Constance l'autorité sur la Bretagne en tant qu'héritière légitime de son père, situation appuyée par le fait qu'elle ait donné naissance à un fils (Pollock, Mélissa, " Duchesses and devils... ", art. cité).

49. WERNER, Karl Ferdinand, "Les femmes, le pouvoir et la transmission du pouvoir ", dans Rouche, Michel et Heuclin, Jean (dir.), La femme au Moyen Âge, Maubeuge, Ville de Maubeuge, 1990, p. 365-381, p. 367-369. 
bien l'héritière du duché de Bretagne. De ce fait, le pouvoir lui est reconnu après la mort de son époux.

L'absence explicite de la qualité en laquelle Constance gouverne doit être soulignée. Judith Everard se demande si elle agit en tant que régente ou en tant qu'héritière ${ }^{50}$. De fait, ce n'est jamais précisé. Si nous prenons le problème à l'envers, et que nous nous intéressons à l'absence de mention de son statut pendant sa période de gouvernement, c'est peut-être parce qu'il n'était pas nécessaire de le spécifier. Constance, fille de duc de Bretagne natif, épouse de duc de Bretagne étranger, et mère d'un duc de Bretagne en devenir, n'a peut-être tout simplement pas eu besoin de produire un statut pour justifier sa place de détentrice de l'autorité ducale. De par tous les éléments qu'elle regroupe, elle était sans doute la personne toute désignée pour assurer le gouvernement de la Bretagne en cette période.

Certains éléments nous l'indiquent. Par exemple, pour deux chartes dont les donateurs mentionnés sont anglais ${ }^{51}$, l'intervention de Constance se justifie par le fait que ces donateurs ont eux-mêmes, antérieurement, bénéficié d'une donation de Conan IV. La duchesse se présente ainsi comme porteuse des octrois effectués par son père, et en tant qu'héritière, elle bénéficie de l'autorité nécessaire pour les confirmer. Elle agit donc en qualité de gouvernante, dans la lignée de ses prédécesseurs. Le fait d'être une femme n'est pas contraignant.

On observe pourtant qu'elle ne se dégage pas de ses devoirs féminins. Constance émet six chartes d'œuvres de foi ${ }^{52}$. Le lien entre la femme et le sacré est fort, et elle détient la responsabilité du salut de l'âme depuis les premiers temps du christianisme ${ }^{53}$. La veuve doit en effet entretenir la memoria de son époux, et également de la famille de ce dernier ${ }^{54}$. Geoffroy est le premier bénéficiaire de ces chartes, puisqu'il est présent dans cinq d'entre elles ${ }^{55}$. Deux des enfants de Constance sont également présents dans la liste des bénéficiaires : Arthur et Mathilde ${ }^{56}$. La femme est liée au sacré dans son rôle de mère. Elle ne doit pas seulement enseigner à ses

50. Everard, Judith Ann, Brittany and the Angevins ..., op. cit., p. 149.

51. $\mathrm{C} 19 ; \mathrm{C} 72$.

52. C4; C17; C20; C31; C38; C72.

53. Livingstone, Amy, "Extraordinairement... ", art. cité, p. 16.

54. JoYE, Sylvie, "Les élites féminines au haut Moyen Âge, historiographie ", dans Les Élites dans le haut Moyen Âge VIe-XII siècle, 2003, p. 9.

55. C17 : Constance fait une donation de sel à l'hôpital Saint-Jean d'Angers pour la mémoire de Geoffroy; C20 : elle fait don d'un terrain et de droits à l'abbaye de Saint-Gildas de Rhuys pour son âme, celles de son mari, de son père et de sa fille Mathilde; C31 : il s'agit d'une autre donation en faveur de l'hôpital Saint-Jean d'Angers pour Geoffroy; C38 : cession de l'île de Brême à l'abbaye du Buzay pour la célébration de son anniversaire, ceux de son père et de son mari, et également pour que les moines prient pour la prospérité de son fils, Arthur; C72 : confirmation d'une cession successive d'une terre que Conan IV avait cédée à Warin de Bassingbourn, qui lui-même l'avait donnée à l'abbaye de Sawtry, en mémoire du père de Constance et de son époux, et pour son âme et celle de son fils.

56. C20; C38; C72. Voir note 20 pour le détail. 
enfants la défense de l'Église et les encourager à faire des donations à des établissements ecclésiastiques, elle doit aussi s'assurer de leur salut ${ }^{57}$. Trois chartes sont en outre émises au bénéfice spirituel de Conan $\mathrm{IV}^{58}$, le père de Constance. Le rôle pieux de la femme ne se limite pas à ses enfants, son époux et la famille de son époux. Il s'étend également à sa propre famille, ce qui permet de réanimer et de rappeler les liens qui les unissent. Pour Constance, il s'agit également d'un moyen de rappeler qu'elle est la fille et l'héritière du duc précédent. Ce dernier est, dans les chartes de cette catégorie, systématiquement associé à Constance et Geoffroy. Cette association souligne la transmission linéaire du pouvoir : Geoffroy détient le pouvoir de par son mariage avec Constance qui, elle, a hérité du pouvoir de son père. Elle se glisse comme l'intercesseur de la transmission de l'autorité ducale entre son père et son époux, puis comme héritière légitime de Conan IV.

Les œuvres de foi constituent une pratique récurrente des corpus traités, mais pas systématique. Ces œuvres prennent des formes qui ne varient pas selon le temps : la prière pour l'âme, l'anniversaire et la mémoire. Dans la majorité des cas, ces œuvres ont pour bénéficiaires les commanditaires et les membres de leur famille. Le corpus de Constance présente cependant une originalité, puisqu'il comprend six œuvres de foi pour un total de soixante-dix-sept actes, ce qui, proportionnellement et en valeur absolue, est plus élevé que dans tous les autres corpus. Constance est la seule femme gouvernant seule la Bretagne dans les corpus que nous avons étudiés et présente de ce fait une situation spécifique en ce qui concerne les œuvres de foi. Il semble que, d'une part, elle use de ses capacités gouvernementales pour agir davantage au bénéfice du salut des membres de sa famille et, d'autre part, qu'elle utilise les œuvres de foi pour rappeler sa légitimité comme porteuse de l'autorité ducale. Le gouvernement du duché ne lui fait donc pas abandonner ce devoir féminin, mais bien au contraire, elle le remplit plus encore et le met au service de l'affirmation de son autorité ducale.

Constance de Bretagne dispose en effet ainsi d'une triple légitimité dans son gouvernement du duché. De ces trois légitimités, elle décide de souligner la première dans sa titulature, ce que l'on peut expliquer de la manière suivante : elle détient son autorité sur la Bretagne par son père, soit par un duc d'origine bretonne. Il est intéressant de souligner ce rappel de légitimité effectué dans la titulature (voir tableau en annexe). Cela constitue une pratique récurrente, au vu du nombre de chartes concernées par la mention " fille du comte Conan $" 59$. En soulignant sa filiation avec Conan IV, Constance affirme ses droits et se présente constamment comme l'héritière

57. LivingSTONE, Amy, Out of love for my kin : aristocratic family life in the lands of the Loire, 1000-1200, Ithaca, Cornell University press, 2010, p. 31-34.

58. C20; C38; C72.

59. C3; C11; C12; C13; C14; C15; C16; C17; C18; C19; C20; C25; C26; C27; C29; C30; C31; C32; C33; C36; C37; C38; C39; C40; C41; C43; C45; C52; C53; C54; C55; C60; C61; C62; C66; C67; C69; C70; C72. 
de son père ${ }^{60}$. Elle ne se présente pas comme étant l'épouse de Geoffroy dans ses chartes, car ce dernier, bien qu'ayant été accepté dans le duché, est d'origine étrangère et est duc de Bretagne de par son mariage, et non par droit de naissance. Quant à Arthur, il n'est encore qu'un enfant et, bien qu'il symbolise l'espoir des Bretons, il n'est pas encore entré dans la sphère du politique.

\section{Un comportement implicite d'interrègne}

Pourtant, Arthur est au cœur de la conception que Constance a de son propre gouvernement. De nombreux indices laissent penser qu'elle le prépare à lui succéder et qu'elle gouverne au nom de son fils mineur ${ }^{61}$.

Le prénom que Constance a choisi pour son fils le prédispose à sa succession. La figure emblématique du roi Arthur a été récupérée par Henri II. Amaury Chauou explique que le roi anglais souhaite se doter d'" un passé conquérant et légitimer son avènement au trône ${ }^{62}$ ». L'objectif est d'entrer en concurrence avec la sacralisation de la dynastie capétienne par le biais de Charlemagne. Ce nouveau culte du roi Arthur est appuyé par de prétendues découvertes. En premier lieu, l'épée du roi Arthur, nommée Excalibur, est trouvée à Glastonbury, transformé en sanctuaire de la royauté anglaise. Le tombeau d'Arthur est ensuite exhumé en $1191^{63}$. La légende arthurienne connaît, à cette période, un véritable regain d'intérêt et un grand succès. Cette instrumentalisation est renversée par Constance. Si Henri II réinvestit la figure arthurienne pour en faire un roi protecteur de son peuple, alors le nouveau-né symbolise l'espoir chez les Bretons luttant pour leur indépendance $^{64}$, ce qui apparaît comme un défi aux yeux d'Henri II. En l'appelant ainsi, Constance a donné une certaine puissance et une certaine valeur à la future titulature de son fils. En sélectionnant ce prénom, elle lie son fils à une parenté et à des honneurs ${ }^{65}$. En effet, les noms identifient les groupes de parenté et appartiennent à des patrimoines familiaux ${ }^{66}$. Mais Constance n'appelle pas son fils Alain, Conan ou encore Hoël, des prénoms répandus chez les ducs bretons du Moyen Âge central, et qui appartiennent bien à

60. Borgnis-DESBordes, Éric, Constance de Bretagne..., op. cit., p. 297. L'auteur observe également que Constance affirme ses droits héréditaires dans ses actes par le rappel de la filiation paternelle.

61. Borgnis-DesBordes, Éric, Arthur de Bretagne..., op. cit., p. 154.

62. Chaud, Amaury, L'idéologie Plantagenêt : royauté arthurienne et monarchie politique dans l'espace Plantagenêt, XII ${ }^{e}$-XIII ${ }^{e}$ siècles, Rennes, Presses universitaires de Rennes, 2001, p. 280. Voir aussi Coumert, Magali et TÉTrel, Hélène (dir.), Histoires des Bretagnes. 1. Les mythes fondateurs, Brest, CERBC-UBO, 2010.

63. Ibid., p. 258.

64. Aurell, Martin, L'empire des Plantagenêt..., op. cit., p. 220-221. Le défi est d'autant plus grand qu'Henri II a insisté pour que l'enfant soit nommé après lui (PoLLOCK, Mélissa, "Duchesses and devils...", art. cité).

65. Livingstone, Amy, Out of love for my kin..., op. cit., p. 158-162.

66. Le JAN, Régine, Femmes, pouvoir et société dans le haut Moyen Âge, Paris, Nouveau monde, 2013, p. 224. 
un patrimoine ducal. Le prénom d'Arthur donne au futur duc une valeur symbolique qui relève du mythe, accordant à la future titulature du duc une dimension supérieure, presque héroïque et protectrice. Arthur dispose ainsi, dès sa naissance, d'une légitimité très forte, comprise et valorisée par les Bretons ${ }^{67}$.

Les $\mathrm{XII}^{\mathrm{e}}$ et XIII ${ }^{\mathrm{e}}$ siècles sont marqués par les premières expériences de régence. L'absence des souverains ou leur minorité entraînent la mise en place d'une délégation de leur pouvoir, assurée par un ou plusieurs individus. Conan III (1112-1148), devenu duc de Bretagne à un jeune âge, gouverne ainsi le duché en co-régence avec sa mère, Ermengarde, pendant environ une trentaine d'année ${ }^{68}$. Au milieu du XII ${ }^{\mathrm{e}}$ siècle, Louis VII (11371180) confie la régence à Suger, assisté du comte de Vermandois, avant de partir pour la seconde croisade. Philippe Auguste fait de même avant de partir pour la troisième croisade, puisqu'il désigne dans son testament de 1190 Adèle de Champagne, veuve de Louis VII, et l'archevêque de Reims comme régents. Au siècle suivant, Blanche de Castille assure la régence pendant la minorité de son fils Louis IX (1226-1270) et également lorsque celui-ci part à son tour pour la septième croisade ${ }^{69}$. La régence est donc employée pour éviter la vacance du pouvoir, et elle est attribuée à des individus des deux sexes.

Le statut de Constance demeure cependant incertain puisqu'il n'est jamais évoqué. Dans ses actes, il n'est jamais écrit qu'elle exerce le pouvoir au nom d'un autre individu, ou que son autorité lui a été déléguée par un tiers. Son gouvernement ne dure pourtant qu'un peu moins d'une quinzaine d'années : pendant la minorité d'Arthur. Il s'agit là d'un comportement d'interrègne, qui n'est pas encore institutionnalisé en régence : exercer l'autorité ducale jusqu'à ce que son fils soit en âge de le faire. Pour autant, le fait-elle de par son statut d'héritière? Il est certain que ce statut lui apporte une légitimité dans ce rôle, tout comme son statut de mère d'Arthur. Elle s'inscrit dans une dynastie qui ne saurait perdurer sans sa présence, et

67. La question du prénom de Constance peut également être soulevée. Il a été attribué à la tante de Constance, sœur de son père Conan IV. Quelques homonymes sont également à relever en dehors de la famille ducale. Constance d'Arles est la troisième épouse de Robert le Pieux (Theis, Laurent, Robert le Pieux, le roi de l'an mil, Paris, Perrin, 2008 (rééd.). L'auteur considère d'ailleurs que ce prénom rappelle l'empire romain. Constance d'Arles est par ailleurs la petite-fille du comte d'Angers Foulques le Bon (p. 131-133). Elle est donc d'ascendance angevine, comme l'était Ermengarde, épouse d'Alain IV et fille de Foulques IV le Réchin (Livingstone, Amy, " Extraordinairement... ", art. cité, p. 9). Contemporaine de Constance de Bretagne, Constance de Hauteville est la fille posthume du roi de Sicile d'origine normande Roger II. Elle a épousé Henri VI du Saint-Empire qui a conquis la Sicile en son nom. Peu avant sa mort, Constance confie le soin de s'occuper de son fils Frédéric au pape Innocent III et au chancelier de Sicile (KANTOROWICZ, Ernst, L'empereur Frédéric II, Paris, Gallimard, 1927 (rééd.), p. 18-20 et 33-34).

68. Livingstone, Amy, "Extraordinairement... ", art. cité, p. 8.

69. CORVISIER, André, "Pour une enquête sur les régences ", Histoire, économie et société, 2002, vol. 21 , n ${ }^{\circ} 2$, p. 206-221, p. 212. 
dont elle assure la succession. De plus, la duchesse adopte cette posture de dépositaire du duché. Elle ambitionne de léguer le duché, intact, à son fils ${ }^{70}$.

Un autre volet de la politique de Constance permet de préciser la conception qu'elle a de son propre pouvoir. Comme le souligne Sylvie Joye, le rôle de la mère s'accroît à partir du Ix ${ }^{\mathrm{e}}$ siècle, puisqu'elle permet l'accès " aux pouvoirs inhérents de la très haute noblesse et est un marqueur de l'appartenance de l'enfant à une élite " ${ }^{71}$. Ainsi, la mère lie son fils au pouvoir, et l'y associe de telle sorte qu'il ne peut être contesté. Elle garantit ainsi la succession dynastique. Pour ce faire, elle prépare Arthur au pouvoir en l'associant à son gouvernement. Constance a par exemple émis six chartes avec Arthur ${ }^{72}$. La duchesse prévoit de se retirer pour laisser la place à Arthur. Animée d'une forte conscience dynastique, Constance assure le lien jusqu'à ce que son fils soit en âge de gouverner. Il semble en effet qu'en 1200, elle se soit retirée de la vie politique, laissant le gouvernement à Arthur.

\section{Le duché au cœur du conflit entre Plantagenêts et Capétiens}

Si l'autorité de Constance n'est pas contestée au sein même du duché, ce n'est pas le cas en dehors de ce dernier. La période est ainsi propice à raviver les conflits entre les Angevins et Philippe-Auguste.

Henri II parvient en effet à intégrer la Bretagne à l'espace Plantagenêt ${ }^{73}$, ce qui renforce la cohérence géographique de ses territoires français, formant un véritable bloc à l'ouest du royaume ${ }^{74}$. Cet agglomérat de territoires est en partie constitué grâce aux alliances matrimoniales, stratégie appliquée à la Bretagne ${ }^{75}$. Cette situation permet de rétablir la traditionnelle subordination du duc de Bretagne au duc de Normandie ${ }^{76}$. L'intégration du

70. Selon Judith Everard, Constance évite " d'aliéner les terres et les revenus ducaux ", afin de ne pas effriter les efforts réalisés par Conan IV et Geoffroy (EvERARD, Judith Ann, Brittany and the Angevins..., op. cit., p. 151).

71. JoYE, Sylvie, "Les élites féminines... ", art. cité, p. 8.

72. C42; C43; C44; C46; C48; C51.

73. La Bretagne est cernée, au nord, à l'est et au sud, par des territoires sous domination Plantagenêt. Franck Bouscau explique qu'elle est encerclée par la puissance « émergente » d'Henri II. Voir BousCAU, Franck, " Réflexions sur les aspects juridiques de la succession à la couronne ducale ", dans QuAGHEBEuR, Joëlle et SolEIL, Sylvain (dir.), Le pouvoir et la foi au Moyen Âge en Bretagne et en Europe de l'Ouest. Mélanges en mémoire du professeur Hubert Guillotel, Rennes, Presses universitaires de Rennes, 2010, p. 375-389, p. 377-378.

74. Il est à noter que la cohérence géographique des territoires Plantagenêts n'est nullement associée à une cohérence politique et institutionnelle.

75. Les alliances matrimoniales sont, pour Henri II, un outil d'extension de son autorité. Ses fils sont habilement mariés à des héritières, comme Henri le Jeune. En 1158, une alliance est négociée entre ce dernier et la fille de Louis VII, Marguerite. Elle apporte dans sa dot le territoire du Vexin (BouRNAZEL, Éric, "La royauté féodale en France et en Angleterre ( $\mathrm{X}^{\mathrm{e}}$-XIII ${ }^{\mathrm{e}}$ siècles)", dans BouRnAZEL, Éric et PoLY, Jean-Pierre (dir.), Les féodalités, Paris, Presses universitaires de France, 1998, p. 389-513, p. 460-461).

76. Comme l'a justement rappelé John Le Patourel, il s'agit pour Henri II " d'un élément de son héritage à rétablir et à garder " (LE PATOUREL, John, " Henri II Plantagenêt..., art. cité, 
duché sécurise également le reste des territoires angevins ${ }^{77}$ et assure une position stratégique dans le réseau des voies commerciales maritimes au sein de l'espace Plantagenêt ${ }^{78}$. Cette nouvelle possession permet enfin à Henri II de préparer sa succession ${ }^{79}$, en pourvoyant son fils d'un territoire assuré.

Tandis qu'Henri II réunit sous son autorité - directe ou indirecte - la moitié du royaume de France, Philippe-Auguste entreprend de reprendre possession des espaces qui semblent lui échapper. La Bretagne fait partie de ces territoires que les deux souverains se disputent. Elle est tombée sous la coupe indirecte de l'Angevin, mais le roi capétien tente de la placer sous sa propre autorité directe.

Entre les années 1187 et 1189, Constance agit de telle sorte qu'aucun reproche ne peut lui être fait de la part des deux souverains, elle conserve ainsi la garde de l'héritier ${ }^{80}$. Elle maintient les officiers placés par les Plantagenêts $^{81}$. À la mort d'Henri II, en 1189, son fils Richard Cour de Lion (1189-1199) lui succède. L'année suivant son accession au trône, il part pour la croisade, permettant à Constance de gouverner sans entrave. Sur la route pour la Terre Sainte, le nouveau roi anglais s'arrête à Messine et fiance Arthur à la fille de Tancrède de Lecce ${ }^{82}$. À cette occasion, il nomme le duc de Bretagne son héritier au trône, écartant ainsi de la succession Jean, son frère cadet $^{83}$.

p. 99-100). L'intégration de la Bretagne est double : il s'agit d'une part d'une intégration par alliance, puisque l'héritière du duché épouse le fils du roi anglais. D'autre part, l'intégration du duché est vassalique, puisque le duc de Bretagne, Geoffroy II, doit rendre hommage à son frère Henri le Jeune en tant que duc de Normandie (CHÉDEVILLE, André et Tonnerre, Noël Yves, La Bretagne féodale..., op. cit., p. 90).

77. EvERARD, Judith Ann, Brittany and the Angevins..., op. cit., p. 35.

78. EvERARD, Judith Ann, " Le duché de Bretagne et la politique Plantagenêt aux XII ${ }^{\mathrm{e}}$ et $\mathrm{XIII}^{\mathrm{e}}$ siècles : perspective maritime ", dans AurELl, Martin et TonNERRE, Noël Yves (dir.), Plantagenêts et Capétiens : confrontations et héritages, Turnhout, Brepols, 2006, p. 193211, p. 193.

79. Madeline, Fanny, "L'empire des Plantagenêts. Espace féodal et construction territoriale ", Hypothèses, n 1, 2007/11, p. 239-252, p. 242.

80. Borgnis-DesBordes, Éric, Arthur de Bretagne..., op. cit., p. 130-131.

81. Il s'agit d'une forme de " respect de la suzeraineté Plantagenêt " pour Yannick Hillion (HILlion, Yannick, "La Bretagne et la rivalité... ", art. cité, p. 116-118).

82. Ibid., p. 116-118.

83. Chauou, Amaury, L'idéologie Plantagenêt..., op. cit., p. 258. 
Constance doit également épouser ${ }^{84}$, en 1188 ou $1189^{85}$, l'homme que le Plantagenêt lui a choisi ${ }^{86}$, l'un de ses fidèles, Ranulf de Chester, alors âgé de 19 ou 20 ans $^{87}$. Il s'agit du second plus grand possessionné en Angleterre, et il possède également des territoires en Normandie ${ }^{88}$. Leur union semble distante : ils n'attestent pas mutuellement leurs chartes. Ranulf, quant à lui, porte bien le titre de duc de Bretagne, mais ne semble pas gouverner en tant que tel ${ }^{89}$.

Lorsque Richard rentre en Europe, une rencontre est organisée en mars 1195 pour réconcilier les deux époux, Ranulf et Constance. Une seconde entreprise de ce type a lieu en $1196^{90}$. Cette même année, Constance est convoquée en Normandie par le roi anglais. Sur le chemin, elle est emprisonnée par son époux et retenue dans son château de Saint-James de Beuvron ${ }^{91}$. Le Plantagenêt demande aux barons du duché de lui remettre Arthur ${ }^{92}$, ce qu'ils refusent. Les détails des événements qui suivent ne nous sont que partiellement connus. Il semble que, dans un premier temps, une réunion a lieu à Saint-Malo-de-Beignon entre les barons, les ecclésiastiques bretons et Arthur. Tous promettent fidélité à Arthur en échange de sa promesse de ne pas conclure de paix séparée avec Richard sans les consulter $^{93}$. Puis, le jeune duc étant menacé, il est emmené par Ghétenoc (désigné évêque de Vannes par Geoffroy en 1182 puis responsable de l'éducation

84. SANTINELli, Emmanuelle, Des femmes éplorées? Les veuves dans la société aristocratique du haut Moyen Âge, Villeneuve d'Ascq, Presses universitaires du Septentrion, 2003, 414 p. L'auteure de l'ouvrage constate déjà qu'au haut Moyen Âge, les veuves sont potentiellement convoitées pour le pouvoir et l'influence qu'elles peuvent exercer et pour les biens qu'elles possèdent (p. 249). Notons que Constance, veuve de Geoffroy II, se remarie deux fois.

85. Le mariage entre la duchesse et son deuxième époux aurait eu lieu, selon Éric Borgnis-Desbordes, du vivant d'Henri II (BORGNIS-DESBORDEs, Éric, Constance de Bretagne..., op. cit., p. 303).

86. Carole Avignon souligne que Richard Cœur de Lion, en fiançant Arthur et en assurant le remariage de Constance, assume son rôle de seigneur d'un vassal décédé selon le droit féodal. Les ducs de Normandie instrumentalisent cette pratique, pour récompenser leurs fidèles vassaux par exemple (BILloré, Maïté, De gré ou de force. L'aristocratie normande et ses ducs (1150-1259), Rennes, Presses universitaires de Rennes, 2014, p. 205-207). Selon Fanny Madeline, cette pratique est intégrée au droit féodal des Normands d'Angleterre. Le comportement de Richard envers Constance et Arthur n'est donc pas isolé mais bien conforme à sa politique de contrôle des alliances matrimoniales.

87. Jones, Michael et EvERARD, Judith Ann, The charters..., op. cit., p. 99.

88. Hillion, Yannick, " La Bretagne et la rivalité... ", art. cité, p. 114.

89. JonEs, Michael, "La vie familiale... ", art. cité, p. 353-354. Ranulf adopte immédiatement le titre de dux Britannie et comes Cestrie et Richemundie sans pour autant l'employer systématiquement. Il n'y a pourtant qu'une seule trace de l'action commune de Constance et Ranulf, à l'occasion d'une revendication des moines de Fougères auprès de l'évêque de Londres. Les chartes publiées à cette occasion l'ont en revanche été séparément (BorGNISDesBordes, Éric, Constance de Bretagne..., op. cit., p. 310-311).

90. Borgnis-Desbordes, Éric, Arthur de Bretagne..., op. cit., p. 162-165.

91. EVERARD, Judith Ann, Brittany and the Angevins..., op. cit., p. 160.

92. Petit-Dutaillis, Charles et Genêt, Jean-Philippe, La Monarchie féodale en France et en Angleterre, $X^{e}$-XIII siècle, Paris, A. Michel, 1971., p. 171.

93. Jones, Michael et EvERARD, Judith Ann, The charters..., op. cit., p. 109. 
d'Arthur ${ }^{94}$ ) pour être protégé par Philippe Auguste ${ }^{95}$ à Paris où il est élevé avec le fils de ce dernier. Mécontent, Richard envahit la Bretagne qu'il ravage avec violence ${ }^{96}$. Il utilise en outre les moyens de pression qui sont à sa disposition, puisque de nombreux nobles de Bretagne possèdent des terres en Normandie et en Angleterre ${ }^{97}$. Une paix est finalement signée en 1196 ou 1197 : Constance est relâchée mais doit se soumettre et suivre les conseils du roi d'Angleterre dans son gouvernement ${ }^{98}$. Les rebelles sont pardonnés et peuvent librement servir Constance, tandis que des terres sont restaurées. Le tout se fait en échange d'un serment de respecter cette paix ${ }^{99}$. Les années qui suivent semblent être relativement calmes, à l'exception de la confiscation de l'honneur de Richemont par le Plantagenêt, en 1198 ou 1199.

Entre-temps, Constance contracte un troisième mariage. Son deuxième époux, Ranulf de Chester, n'est pas décédé, et nous ignorons tout des circonstances de l'annulation de leur mariage ${ }^{100}$. En septembre ou en octobre 1199, quelques mois après le décès de Richard Cœur de Lion, Constance se marie donc pour la troisième fois avec Guy de Thouars, qui est probablement choisi par Jean sans Terre ${ }^{101}$. De cette union naissent trois filles : Alix ${ }^{102}$, Marguerite et Catherine.

Il faut également noter que la garde des deux enfants du défunt Geoffroy II est disputée entre les rois capétien et anglais. Finalement, Aliénor est confiée à Henri II et se rend en Angleterre ${ }^{103}$ (retenue captive, elle meurt en 1241 à Bristol ${ }^{104}$ ), tandis que dans un premier temps Constance conserve

94. Tourault, Philippe, Les ducs et duchesses..., op. cit., p. 100 et 104.

95. ChÉDeville, André et TonNerre, Noël Yves, La Bretagne féodale..., op. cit., p. 98.

96. HiLlon, Yannick, « La Bretagne et la rivalité... », art. cité, p. 119.

97. Aurell, Martin, L'empire des Plantagenêt..., op. cit., p. 223. Certains barons bretons (les Fougères, les Vitré, les Dol, les Dinan ou encore les Mayenne) sont possessionnés outre-Manche car leurs ancêtres ont participé à la conquête anglaise.

98. HiLlion, Yannick, « La Bretagne et la rivalité... », art. cité, p. 120.

99. EVERARD, Judith Ann, Brittany and the Angevins..., op. cit., p. 165-166.

100. Il est fort probable que le mariage entre Constance et Ranulf de Chester ait été annulé, puisqu'aucun des deux époux n'est décédé. Les sources demeurent silencieuses quant à cette affaire. Il est cependant possible de supposer que la non cohabitation des époux, voire la non consommation du mariage, soit le motif retenu pour l'annulation.

101. EVERARD, Judith Ann, Brittany and the Angevins..., op. cit., p. 171-172. Éric BorgnisDesbordes propose la théorie d'un mariage d'" amour ", en soulignant que Guy est un cadet de famille et un baron d'un rang nettement inférieur au sien (BORGNIS-DESBORDES, Éric, Constance de Bretagne..., op. cit., p. 422).

102. Alix, représentante de la dynastie ducale à la suite du décès d'Arthur, épouse en 1213 Pierre de Dreux, parent fidèle de Philippe Auguste (AuRELL, Martin, L'empire des Plantagenêt..., op. cit., p. 219). Cette alliance matrimoniale marque la victoire du capétien dans la lutte d'influence sur la Bretagne.

103. Aliénor aurait été confiée à la garde de sa grand-mère, Aliénor d'Aquitaine. Elle a fait l'objet d'une prospection matrimoniale agencée par Richard Cœur de Lion, qui avait prévu de la marier avec le fils du duc d'Autriche Léopold (BORGNIs-DESBORDES, Éric, Constance de Bretagne..., op. cit., p. 307 et 319).

104. Pocquet Du Haut-Jussé, Barthélemy Amédée, "Les Plantagenets et la Bretagne ", Annales de Bretagne, 1946, vol. 53, nº 2, p. 1-27, p. 14. 
la garde d'Arthur ${ }^{105}$. La garde de l'héritier semble être primordiale ${ }^{106}$. Celui qui détient Arthur détient également le pouvoir sur la Bretagne. Une telle conception indique que Constance n'est pas reconnue comme duchesse régnante en son propre nom.

La mort de Richard I ${ }^{\mathrm{er}}$, en 1199, entraîne une nouvelle guerre de succession, mais cette fois à l'échelle de l'espace Plantagenêt. Le roi défunt n'a laissé aucun enfant et a désigné, quelques années auparavant, le jeune duc de Bretagne comme étant son héritier. Le duc en question a quitté la cour de France cette même année. Jean sans Terre (1199-1216) s'oppose à son neveu. Deux règles successorales s'opposent : la prérogative d'aînesse par représentation ${ }^{107}$ (Arthur est le fils unique de Geoffroy, frère aîné de Jean) et le droit de viage ou de retour, favorable au frère cadet du défunt. La Bretagne, le Maine, l'Anjou, la Touraine et le nord du Poitou soutiennent Arthur ${ }^{108}$, tandis que l'Angleterre, la Normandie et l'Aquitaine (tout comme Aliénor d'Aquitaine) sont partisans de Jean. Le conflit est militaire, et les deux adversaires cherchent à prendre des territoires. Afin de se doter de légitimité, Arthur se rend à Angers pour revendiquer le patrimoine angevin $^{109}$, puis fait l'objet d'une seconde cérémonie à Tours. Jean, quant à lui, est investi duc de Normandie et couronné roi d'Angleterre. Philippe Auguste participe au conflit et prend le parti d'Arthur, qui lui jure fidélité et lui rend hommage. Les tentatives de négociation échouent, et les trois hommes sont en guerre ${ }^{110}$. Sur le terrain militaire, Philippe Auguste rencontre finalement quelques difficultés, qui semblent le mener au traité du Goulet, signé le 22 mai $1200^{111}$. Par ce traité, le roi français reconnaît Jean comme l'héritier de Richard et Arthur comme l'héritier de la Bretagne. Ce dernier doit rendre hommage à Jean pour son duché ${ }^{112}$, et est placé sous la tutelle de Philippe Auguste ${ }^{113}$. Cette configuration permet au roi de France d'avoir la Bretagne sous sa responsabilité ${ }^{114}$. Cette situation contraint-elle Constance à se retirer du pouvoir? Ou a-t-elle considéré que sa période de gouvernement était achevée?

105. ChÉDEvilLE, André et TonNerRe, Noël Yves, La Bretagne féodale ..., op. cit., p. 96.

106. Il semblerait que Constance ait dissimulé son enfant à Henri II lors de sa venue à Nantes, afin d'éviter qu'il ne s'en empare (Borgnis-DesBordes, Éric, Constance de Bretagne..., op. cit., p. 302).

107. Borgnis-DesBordes, Éric, Constance de Bretagne..., op. cit., p. 385.

108. AURELL, Martin, L'empire des Plantagenêt..., op. cit., p. 223.

109. PocQuet Du hauT-JusSÉ, Barthélemy Amédée, "Les Plantagenets... ", op. cit., p. 13-14.

110. Jones, Michael et EvERARD, Judith Ann, The charters..., op. cit., p. 109-110.

111. Borgnis-Desbordes, Éric, Constance de Bretagne (1161-1201)..., op. cit., p. 428.

112. EvERARD, Judith Ann, Brittany and the Angevins..., op. cit., p. 173

113. Hillion, Yannick, "La Bretagne et la rivalité... ", art. cité, p. 125.

114. BalDwIN John, Philippe Auguste, Paris, Fayard, 1991 (rééd.), p. 308. L'auteur explique que Philippe Auguste prend le parti du candidat jugé le plus jeune et le plus faible, Arthur, contre le candidat le plus fort et le plus dangereux. Cette situation permet au capétien d'avoir une main sur les affaires du duché, comme la confirmation de dons réalisés par Arthur auprès de ses partisans, en 1199 (p. 133). 
Si Constance ne rencontre pas de difficultés à l'intérieur de son duché, où elle est bien acceptée par les barons bretons qui la traitent comme leur souveraine, il n'en est pas de même à l'extérieur du duché où elle doit faire face aux contestations. Si la garde d'Arthur lui est contestée par les rois étrangers, c'est probablement parce qu'elle est une femme. Il est vrai qu'en période de guerre et d'absence, les femmes sont autonomes par rapport à leurs enfants mineurs ${ }^{115}$. Mais ici, il ne s'agit pas d'une substitution ponctuelle, le duc de Bretagne n'est pas en guerre sur un autre territoire, ni en voyage en dehors du duché, il est décédé. Ce qui place la Bretagne, en apparence, en situation de régence. Ceci est perçu comme un moment de faiblesse par les Plantagenêts et Philippe Auguste, et ils profitent de cette période pour tenter de s'emparer de l'héritier, Arthur, et ainsi de faire passer la Bretagne sous leur domination. Le sexe de Constance entraîne cette compétition entre les rois capétien et anglais. De ce fait, Constance est obligée d'agir avec prudence. Concernant les Plantagenêts, Henri II puis Richard Cœur de Lion, elle se comporte en bonne vassale, afin de conserver la garde d'Arthur et l'indépendance de la Bretagne. Elle ne veut pas leur donner prétexte à une quelconque ingérence dans ses affaires.

L'exercice du pouvoir par Constance en tant que duchesse de Bretagne est attesté. Les sources ne présentent aucune contestation à ce pouvoir, et aucune mention n'est faite d'un débat autour du détenteur du pouvoir en Bretagne suite au décès de Geoffroy. Constance semble s'être imposée naturellement, car la Bretagne n'est pas seulement sa dot, mais également son héritage. En tant qu'enfant unique de Conan IV, elle hérite du duché sans que son genre soit un obstacle à cette succession ${ }^{116}$.

Constance doit à son tour transmettre cet héritage. Les Bretons ne veulent pas de souverains étrangers à la tête de leur duché. Geoffroy a été accepté car Constance se tenait à ses côtés, mais également parce que lui-même a montré cette volonté d'indépendance en tant que seigneur de Bretagne. Cet héritage demeure donc breton, et la garantie de cette indépendance n'est autre qu'un gouvernant breton. La mort de Geoffroy est, pour les souverains étrangers et notamment pour le Plantagenêt, l'opportunité de recouvrer une autorité directe sur le duché. Le barrage à cette entreprise est tout simplement Constance : fille de duc breton, épouse de duc breton (qui est également un Plantagenêt) et mère de duc breton en devenir. Elle est celle qui concentre le plus de légitimité et apparaît donc comme la gouvernante naturelle du duché, permettant à la fois d'assurer la stabilité de la Bretagne et d'éviter qu'elle ne tombe davantage dans le giron Plantagenêt. Cette succession semble être une évidence, par la rapidité et la clarté de sa mise en place. Si Constance devient la gouvernante de

115. Livingstone, Amy, Out of love for my kin..., op. cit., p. 186

116. Franck Bouscau rappelle qu'entre le XII et le XIV siècle, le duché de Bretagne se transmet tel un fief et que les femmes et les enfants ne sont pas exclus de la succession (BousCAU, Franck, " Réflexions sur les aspects... ", art. cité, p. 377). 
Bretagne, c'est parce qu'elle est toute désignée pour accomplir cette tâche. Son sexe ne semble pas avoir été un problème dans le déroulement des événements, car sa naissance et sa filiation sont là pour rappeler qu'elle est un maillon indispensable dans la succession de la dynastie ducale bretonne.

Son sexe féminin est pourtant utilisé par les souverains anglais et français pour justifier leur intervention. Ils tentent ainsi de la verrouiller par le mariage (et la condition d'épouse) et la perte de la tutelle d'Arthur. Affaiblie par son sexe en dehors du duché, sa filiation - bretonne et ducale - a protégé la Bretagne d'une gouvernance étrangère. La conscience dynastique de Constance l'a donc poussée à assurer une période transitoire de gouvernement, à maintenir la lignée entre deux ducs, son mari et son fils. En assumant cette responsabilité temporaire, elle devient actrice du maintien et de la reproduction de la domination masculine ${ }^{117}$. Constance comble une vacance masculine pour assurer la continuité entre deux ducs. Cependant, cette domination masculine est secondaire, et se positionne derrière la domination dynastique et lignagère, dont le maintien est l'objectif premier de la duchesse.

117. BouRDIEu, Pierre, La domination masculine, Paris, Éditions du Seuil, 1998. Si le constat d'une domination masculine est valable pour la période centro-médiévale, cette domination demeure secondaire et insérée dans des groupes sociaux et des structures sociales plus ou moins vastes. La domination sociale s'opère ainsi en priorité selon d'autres facteurs discriminants, parmi lesquels se trouve la filiation. 
La duchesse Constance de Bretagne et le gouvernement en héritage à la fin du XII ${ }^{\mathrm{e}}$ siècle

Annexe - Titulature* de Constance dans les chartes (77 chartes)

\begin{tabular}{|c|c|c|c|}
\hline Titulature de Constance & Chartes concernées & $\begin{array}{c}\text { Nombre } \\
\text { de chartes } \\
\text { concernées }\end{array}$ & Pourcentage \\
\hline $\begin{array}{l}\text { "Constance fille de } \\
\text { Conan " } \\
\text { «Constantia filia Conani " }\end{array}$ & $\mathrm{C} 11$ & 1 & $1 \%$ \\
\hline $\begin{array}{l}\text { "Constance, comtesse de } \\
\text { Bretagne" } \\
\text { "Constantiam comitissam } \\
\text { Britannie" }\end{array}$ & $\mathrm{C} 28$ & 1 & $1 \%$ \\
\hline $\begin{array}{l}\text { "Constance, duchesse de } \\
\text { Bretagne, comtesse de } \\
\text { Richemont" } \\
\text { "Constancia ducissa } \\
\text { Britanniae, comitissa } \\
\text { Richemondiae" }\end{array}$ & $\begin{array}{l}\text { C4; C5; C42; C51; } \\
\text { C59 }\end{array}$ & 5 & $6 \%$ \\
\hline $\begin{array}{l}\text { "Constance, fille du } \\
\text { comte Conan, Duchesse } \\
\text { de Bretagne, Comtesse de } \\
\text { Richemont" } \\
\text { "Constantia, comitis Cona- } \\
\text { ni filia, ducissa Britanniae, } \\
\text { comitissa Richemondiae" }\end{array}$ & $\begin{array}{l}\text { C3; C12; C13; C14; } \\
\text { C15; C17; C18; C19; } \\
\text { C20; C25; C26; C27; } \\
\text { C29; C30; C31; C32; } \\
\text { C33; C36; C37; C38; } \\
\text { C39; C40; C41; C43; } \\
\text { C45; C52; C53; C54; } \\
\text { C55; C60; C61; C62; } \\
\text { C66; C67; C69; C70; } \\
\text { C72 }\end{array}$ & 37 & $48 \%$ \\
\hline $\begin{array}{l}\text { "La fille du comte Conan, } \\
\text { duchesse de Bretagne, } \\
\text { comtesse de Richemont" } \\
\text { "comitis Conani filia } \\
\text { ducissa Britanniae, comi- } \\
\text { tissa Richemondiae" }\end{array}$ & $\mathrm{C} 16$ & 1 & $1 \%$ \\
\hline $\begin{array}{l}\text { "Ma femme Constance" } \\
\text { "Constancia uxor mea" }\end{array}$ & $\mathrm{C} 6 ; \mathrm{C} 10$ & 2 & $3 \%$ \\
\hline $\begin{array}{l}\text { "Ma femme la comtesse } \\
\text { Constance " "C[onstantia] } \\
\text { comitisse uxoris eius " }\end{array}$ & $\mathrm{C} 1$ & 1 & $1 \%$ \\
\hline Aucune & $\mathrm{C} 2 ; \mathrm{C} 8$ & 2 & $3 \%$ \\
\hline Inconnue & $\begin{array}{l}\text { C7; C9; C21; C22; } \\
\text { C23; C24; C34; C35; } \\
\text { C44; C46; C47; C48; } \\
\text { C49; C50; C56; C57; } \\
\text { C58; C63; C64; C65; } \\
\text { C68; C71; C73; C74; } \\
\text { C75;C76;C77 }\end{array}$ & 27 & $35 \%$ \\
\hline
\end{tabular}

* Titulature : titres et affiliations contenus dans l'intitulation des chartes 


\section{RÉSUMÉ}

À la fin du XII ${ }^{\mathrm{e}}$ siècle, le duché de Bretagne se retrouve au cœur du conflit opposant les Plantagenêts aux Capétiens. Henri II, roi d'Angleterre, a intégré le duché breton dans sa zone d'influence en soutenant la reconquête du duché par le duc Conan IV dans un premier temps, et en mariant ensuite l'héritière de ce dernier, Constance, à son propre fils, Geoffroy. À la mort de celui-ci, en 1186, Constance est enceinte d'un fils qui naît l'année suivante. Pendant une quinzaine d'années, Constance gouverne effectivement le duché. Le statut au nom duquel elle assume ce gouvernement n'est pourtant jamais explicité. Agit-elle en tant qu'héritière de son père, en tant que veuve de son défunt mari, en tant que mère du jeune héritier ? Si la documentation demeure silencieuse, il n'en reste pas moins que les comportements de la duchesse nous permettent de donner quelques éléments d'explication. Constance, duchesse de Bretagne, s'inscrit dans une dynastie dont elle assure la continuité, malgré les tentatives répétées des rois anglais et français pour verrouiller son autorité.

\section{ABSTRACT}

At the end of the twelfth century, the duchy of Brittany was deeply involved in the conflict between the Plantagenets and the Capetians. Henry II, King of England, integrated the duchy to his area of influence initially by supporting Duke Conan IV's reconquest of the duchy, and then by marrying the duke's heiress, Constance, to his own son, Geoffroy. When the latter died in 1186, Constance was pregnant with a son who was born in 1187. During about fifteen years, Constance effectively ruled the duchy alone. Yet, by which authority she governed was never made explicit. Was she acting as the heiress of her father, as the widow of her dead husband, or as the mother of the young heir? If extant sources fail to provide an answer to this question, the duchess' behaviour gives us some elements of explanation. Constance, Duchess of Brittany, was part of a dynasty that she sought to perpetuate, despite the repeated attempts by the English and French kings to limit her authority. 